\title{
ESTUDIO DE LA VARIABILIDAD TEMPORAL Y ESPACIAL DE LA PÉRDIDA DE SUELO EN LA DEHESA (EXTREMADURA, SO DE ESPAÑA) MEDIANTE LLUVIA SIMULADA*
}

\author{
A. Ceballos Barbancho (1), S. Schnabel (2) y A. Cerdá (3) \\ (1)- Departamento de Geografía, Universidad de Salamanca. \\ C/ Cervantes, 3. 37001-Salamanca. \\ (2)- Depto. de Geografía y O.T., Univ de Extremadura. \\ Avda. de la Universidad s/n. 10071-Cáceres. \\ (3)- Instituto Pirenaico de Ecología, CSIC. \\ Campus Aula Dei, Apartado 202. 50080-Zaragoza.
}

\begin{abstract}
Resumen: El objetivo principal del presente trabajo es el estudio de la influencia de la variabilidad estacional de las condiciones antecedentes en la pérdida de suelo en un ecosistema adehesado, así como una aproximación a su variabilidad espacial. Para ello se han realizado 40 experimentos de simulación de lluvia en dos situaciones contrastadas: por un lado un ambiente seco, con vegetación degradada, y, por otro lado un ambiente húmedo, con una cobertera vegetal importante.
\end{abstract}

Los resultados indican que las mayores concentraciones de sedimentos se localizan en situaciones de suelos desprotegidos, tanto por el efecto de la sequía como del tránsito del ganado, y que el control del proceso responde más al suministro de material que a la capacidad de transporte de la arroyada. Desde una perspectiva espacial, destaca el contraste entre las bajas pérdidas registradas en los fondos de valle y los valores más altos medidos en las zonas más frecuentadas por el ganado, en donde la degradación de la cubierta vegetal y la remoción del material es continua. Finalmente, aunque desde un punto de vista cuantitativo, la pérdida de suelo en la dehesa es baja, sí lo es desde un punto de vista cualitativo al afectar al horizonte más fértil del perfil edáfico.

Palabras clave: erosión, concentración de sedimentos, estacionalidad, escorrentía, simulación de lluvia, dehesa.

Abstract: The main objective of this paper is to study the influence of the seasonal variability of the antecedent conditions in soil losses in a dehesa ecos-

* Recibido: 16-7-2001. Aceptado: 19-4-2002 
ystem, as well as an approach to its spatial variability. The experimental layout includes forty rainfall simulation experiments carried out in two different situations: first on dry conditions, with degraded vegetation, and, second on wet conditions, with an important vegetation cover.

The results indicate that the highest sediment concentrations are located in situations with bare soils, due to the effect of the drought and the soil removed by grazing animals. The control of the process depends more to the sediment available on the soil surface than to the runoff transport capacity. From an spatial point of view, it highlights the contrast between the low losses registered in the valley bottoms and the highest values measured in the areas more frequented by the livestock, where the degradation of the vegetation cover and the removal of the soil material are continuous. Finally, although the rate of soil loss in the dehesa is low, it is important from a qualitative point of view because it affects to the most fertile horizon in the soil profile.

Key words: soil loss, sediment concentration, season, runoff, rainfall simulation, dehesa.

\section{Introducción}

La dehesa constituye uno de los usos del suelo más frecuentes en el suroeste de la Península Ibérica debido a su gran nivel de adaptación a las condiciones físicas limitantes de esta zona (Ibáñez et al., 1981), hasta tal punto que una dehesa explotada de manera correcta se presenta como el sistema óptimo de utilización de los recursos agrobiológicos en ambientes semiáridos (Gómez Gutiérrez, 1987). Suelen estar asentadas sobre penillanuras con un sustrato geológico de pizarras, granitos y cuarcitas, suelos poco profundos, con escasa materia orgánica y pobres en bases. El régimen pluviométrico oscila entre rasgos semiáridos y subhúmedos, con una precipitación media comprendida entre los 400 y $600 \mathrm{~mm}$, y una acusada irregularidad tanto anual como interanual.

Constituidas por un bosque abierto de quercíneas, la influencia del factor humano es fundamental mediante un triple uso agro-silvo-pastoral de sus recursos, siendo el pastoreo extensivo la práctica más habitual en las dehesas.

Ante la ausencia de estudios sobre los procesos que modelan el medio físico en las dehesas, y debido a su gran importancia ecológica y económica en el suroeste de la Península Ibérica, desde 1990 se están analizando los procesos hidrológicos y erosivos que operan en una pequeña cuenca hidrográfica en ambiente semiárido y representativa del sistema dehesa (Gómez-Amelia y Schnabel, 1992; Schnabel, 1997; Mateos y Schnabel, 1998; Ceballos y Schnabel, 1998; Ceballos, 1999). 
Uno de los principales problemas de las zonas semiáridas es la pérdida de suelo debido a las condiciones climáticas y presión de las actividades antrópicas (United Nations, 1977). Dependiendo de la variabilidad climática y del grado de intensidad del uso del ecosistema, por parte del hombre, variarán las condiciones para la pérdida de suelo.

El objetivo del presente trabajo es estudiar cómo influye la variabilidad de las condiciones antecedentes en la pérdida de suelo en el ecosistema dehesa. Para ello se han seleccionado dos situaciones ambientales muy distintas como consecuencia de la distribución anual de las lluvias y del grado de intensidad del pastoreo: un ambiente denominado seco, con vegetación degradada, y un ambiente denominado húmedo, con vegetación abundante. El uso de un simulador de lluvia, técnica que permite un gran control de las condiciones de la lluvia por el investigador, y la distinción previa de diversas unidades espaciales sobre el terreno han permitido, además del estudio de los contrastes estacionales, una aproximación a la variabilidad espacial de la pérdida de suelo en la dehesa.

\section{Zona de estudio}

La cuenca experimental de Guadalperalón se localiza a $22 \mathrm{~km}$ al noreste de la ciudad de Cáceres (figura 1), siendo representativa de las zonas adehesadas típicas de la mitad occidental de la Península Ibérica. Asentada sobre la superficie de erosión finimiocena de la penillanura cacereña (Gómez-Amelia, 1985), el sustrato geológico está representado en su totalidad por el Complejo Esquisto Grauváquico. Los suelos se encuadran dentro del grupo de los leptosoles y regosoles, con una profundidad muy variable, textura generalmente franca, pobre estructura, alta densidad aparente y baja cantidad de materia orgánica.

El clima es mediterráneo debido a un neto contraste estacional en los valores térmicos y, sobre todo, pluviométricos. La temperatura media anual es de $16^{\circ} \mathrm{C}$, mientras que el total anual de precipitaciones se eleva a $517 \mathrm{~mm}$ (media del período 1907-2000), mostrando una importante variabilidad tanto anual como interanual.

La vegetación potencial de la zona está formada por un encinar de Quercus ilex subsp. ballota, con una densidad que oscila entre los 15 y los 45 pies ha1 , aunque algunos sectores de la cuenca presentan un estadio muy regresivo con el dominio de un matorral abierto de Lavandula stoechas. En los últimos años, la dehesa de Guadalperalón ha sido pastoreada por rebaños de cabras, cerdos, vacas y, principalmente, ovejas, con una carga variable a lo largo del tiempo. 


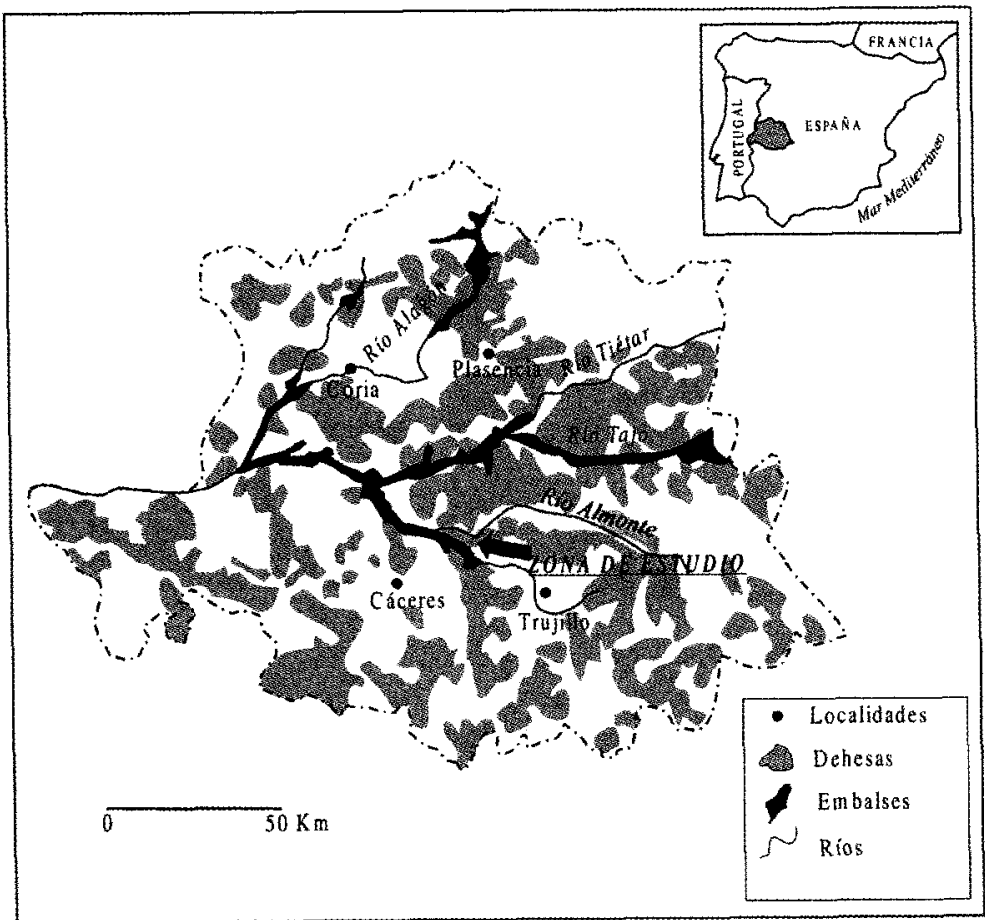

Figura 1. Localización de la zona de estudio y extensión de las zonas adehesadas en la provincia de Cáceres.

\section{Métodos}

El simulador de lluvia utilizado en las experimentaciones es del tipo pulverizador, según el diseño de Cerdà et al. (1997). Básicamente se compone de un equipo de presurización manual de presión previa por aire comprimido, una estructura formada por una plataforma cuadrada de $40 \mathrm{~cm}^{2}$ y 4 apoyos telescópicos con una longitud máxima de $3,45 \mathrm{~m}$, un protector de viento para evitar alteraciones en la dirección de caída de las gotas de lluvia y un juego de boquillas tipo HARDI. Se ha aplicado una presión entre 1,5 y $1,6 \mathrm{~kg} \mathrm{~cm}^{-2}$, que equivalen a una intensidad entre 53 y $55 \mathrm{~mm} \mathrm{~h}^{-1}$, ya que garantiza la distribución más homogénea de la lluvia. La duración de cada experimento ha sido de 1 hora con el objetivo de asegurar la generación de escorrentías estables y observar la evolución de la concentración de sedimentos. Aunque la superficie sobre la que cae la lluvia es de $1 \mathrm{~m}^{2}$, las mediciones se realizan en la parte central de la misma para minimizar el efecto borde (Meyer, 1988). Se han utilizado parcelas móviles de forma circular con un área de $0,24 \mathrm{~m}^{2}$ y un diámetro de $55 \mathrm{~cm}$. 
La realización de cada experimento obedece al siguiente protocolo (Cerdà, 1993 y 1995a; Ceballos, 1999): i) descripción de la superficie del suelo atendiendo a la litología, pedregosidad, agrietamientos, costras, vegetación, hojarasca, situación, uso del suelo, etc.; ii) colocación de la microparcela sobre el terreno; iii) estimación sobre la parcela del porcentaje de cobertura del suelo; iv) medición por gravimetría de la humedad del suelo antes del experimento, calculándose con las mismas muestras tanto la densidad como la porosidad; v) instalación del simulador sobre la parcela, puesta en funcionamiento del mismo y desarrollo propiamente del experimento; vi) finalizado el experimento se procede a la misma sistemática, previa a su inicio, con la descripción de la superficie para ver posibles alteraciones producidas por la lluvia, toma de muestras del suelo para analizar los cambios en la humedad, porosidad y densidad, y finalmente se excava la parte central de la microparcela para dibujar el frente de humedad.

Durante el desarrollo del experimento se miden diversos parámetros hidrológicos como tiempo de encharcamiento, tiempo de escorrentía, escorrentía media, coeficiente de escorrentía, capacidad de infiltración final estable, etc. A efectos del presente trabajo se tomaron muestras de sedimentos al comienzo de la simulación, en la parte media y al final de la misma. Ello ha permitido estudiar la evolución temporal de la concentración media de sedimentos (expresada en $\mathrm{g}^{-1}$ ) durante la lluvia y estimar una tasa de erosión media (expresada en $\mathrm{g} \mathrm{m}^{-2} \mathrm{~h}^{-1}$ ) para cada una de las microparcelas.

La simulación de lluvia se muestra como la técnica más adecuada para la finalidad del presente trabajo, ya que otorga un gran control de las condiciones de la lluvia al propio investigador. Ello permite atribuir las diferencias de los resultados obtenidos a la variabilidad temporal y espacial de las superficies sobre las que se experimenta. Con mediciones de erosión en parcelas bajo lluvia natural es difícil precisar con claridad las diferencias estacionales debido a la gran variabilidad de los eventos lluviosos en zonas semiáridas.

No obstante, la metodología empleada también presenta una serie de limitaciones si se pretende comparar los resultados de las simulaciones con los hallados en parcelas de erosión con lluvias naturales. La energía cinética de la lluvia es baja debido a que la mayor parte de las gotas producidas tienen un tamaño inferior a $1 \mathrm{~mm}$ (Cerdà et al., 1997). La altura de la boquilla impide que las gotas alcancen su velocidad terminal, significando que la capacidad erosiva de las lluvias simuladas es menor a la que produciría una lluvia natural con intensidad de $53,6 \mathrm{~mm} \mathrm{~h}^{-1}$ (Epema y Riezebos, 1983). Por otro lado, el reducido tamaño de las parcelas impide la concentración de flujo superficial, por lo que el grado de velocidad y turbulencia de la escorrentía superficial durante las simulaciones es bajo.

En total se realizaron 40 experimentos divididos en dos series de 20 . La primera se llevó a cabo a finales de mayo de 1995, con un balance pluviométrico de sólo $40 \mathrm{~mm}$ en los tres meses previos a los experimentos y $1,7 \mathrm{~mm}$ en los 15 días anteriores. El 
pastoreo fue continuado durante todo el año, a pesar de la sequía, con una càrga de 1,6 ovejas ha ${ }^{-1}$ en el momento de realizarse los experimentos. Consecuentemente, el pasto estaba bastante degradado y los porcentajes de suelo desnudo eran apreciables. La segunda serie data de mediados de febrero de 1996. Las lluvias correspondientes a los tres meses precedentes se elevaron a $508 \mathrm{~mm}$, con $36 \mathrm{~mm}$ en los 15 días previos. En el momento de realizarse las simulaciones la cuenca llevaba dos meses sin ganado, lo que explica, junto a las abundantes lluvias, la existencia de un porte herbáceo abundante y bastante uniforme en toda la cuenca. Este contraste permitió el estudio de la pérdida de suelo en la dehesa ante condiciones antecedentes muy diferentes.

Para el análisis de la variabilidad espacial de los resultados se utilizaron las mismas unidades que se determinaron para el estudio de la respuesta hidrológica de los suelos de la zona (Cerdà et al., 1998) y que aparecen sintetizadas en la tabla 1.

Tabla 1. Descripción de las unidades espaciales atendiendo a las características edáficas, vegetación y usos del suelo.

\begin{tabular}{|ll|}
\hline Unidad & Descripción \\
\hline $\begin{array}{l}\text { Laderas con pastos } \\
\text { (HG) }(\mathrm{n}=9)\end{array}$ & $\begin{array}{l}\text { Vertientes situadas entre los árboles. Su cubierta de pastos } \\
\text { es muy sensible al clima y pastoreo. Debido a que es la unidad } \\
\text { con mayor extensión en la cuenca en ella se realizaron } 9 \text { experi- } \\
\text { mentos. }\end{array}$ \\
\hline $\begin{array}{l}\text { Fondos de valle con } \\
\text { pasto }(\mathrm{BG})(\mathrm{n}=3)\end{array}$ & $\begin{array}{l}\text { Representan los fondos de valle, caracterizados por suelos } \\
\text { profundos y cobertura herbacea abundante. }\end{array}$ \\
\hline $\begin{array}{l}\text { Áreas bajo arbolado } \\
\text { (TC) }(\mathrm{n}=3)\end{array}$ & $\begin{array}{l}\text { Lugares de las vertientes situados bajo la proyección cle las } \\
\text { copas de } Q \text {. ilex subsp. ballota. Se caracterizan por un horizonte } \\
\text { superficial de hojarasca. }\end{array}$ \\
\hline $\begin{array}{l}\text { Veredas } \\
\text { (ST) }(\mathrm{n}=4)\end{array}$ & $\begin{array}{l}\text { Gran parte de la superficie está ocupada por una franja de } \\
\text { suelo desnudo resultado del constante pisoteo del ganado. }\end{array}$ \\
\hline $\begin{array}{l}\text { Arbustos } \\
\text { (SC) }(\mathrm{n}=2)\end{array}$ & $\begin{array}{l}\text { Vertientes sin arbolado y cubiertas parcialmente por man- } \\
\text { chas de } L \text { stoechas. }\end{array}$ \\
\hline
\end{tabular}

\section{Resultados y discusión}

\subsection{Condiciones antecedentes}

Las tablas 2 y 3 resumen las condiciones antecedentes de las parcelas, tanto en ambiente seco como húmedo, agrupadas en sus respectivas unidades espaciales. Debido a la orientación en sentido meridiano de la cuenca, todas las parcelas selec- 
cionadas presentan una exposición este-oeste con la excepción de las tres localizadas en los fondos de valle (BG), que es norte-sur ante la ausencia de pendientes significativas. La pendiente media de las diversas unidades, excepto los fondos de valle (BG), es muy parecida, con un rango comprendido entre los 11 y $12,6^{\circ}$ (tablas 2 y 3 ). En la parte central del fondo de valle la pendiente es muy baja $\left(2^{\circ}\right)$. Esta circunstancia es significativa, ya que los fondos de valle actúan como zona acumuladora, tanto de la escorrentía como del sedimento producido en las vertientes. Además, en los fondos de valle se localizan los suelos más profundos en contraste con las vertientes. La ausencia de horizontes nos permite asociar su génesis a procesos recientes de erosión acelerada.

Tabla 2. Características de las superficies en el período seco. Promedio por unidades y valor de la media y coeficiente de variación correspondientes al conjunto de la serie $(n=20)$.

\begin{tabular}{|cccccccccccc|}
\hline $\begin{array}{c}\text { Unidad } \\
(\%)\end{array}$ & $\begin{array}{c}\text { Exp } \\
(\%)\end{array}$ & $\begin{array}{c}\text { Pen } \\
(\%)\end{array}$ & $\begin{array}{c}\text { L+M } \\
(\%)\end{array}$ & $\begin{array}{c}\text { P1 } \\
(\%)\end{array}$ & $\begin{array}{c}\text { Hoj } \\
(\%)\end{array}$ & $\begin{array}{c}\text { Vtot } \\
(\%)\end{array}$ & $\begin{array}{c}\text { Aveg } \\
(\mathrm{cm})\end{array}$ & $\begin{array}{c}\text { Pe } \\
(\%)\end{array}$ & $\begin{array}{c}\text { Ps } \\
(\mathrm{cm})\end{array}$ & $\begin{array}{c}\text { Hs } \\
(\%)\end{array}$ & $\begin{array}{c}\text { Den } \\
\left(\mathrm{g} \mathrm{cm}^{3}\right)\end{array}$ \\
\hline 1-HG & 131 & 12,6 & 9,2 & 3,2 & 15,8 & 28,2 & 2,0 & 7,6 & 36,8 & 1,87 & 1,29 \\
2-BG & 240 & 2,0 & 12,3 & 60,0 & 13,3 & 85,7 & 15,3 & 3,3 & 100,0 & 8,15 & 1,00 \\
3-TC & 72 & 11,3 & 3,3 & 7,0 & 60,0 & 70,3 & 3,3 & 1,7 & 43,3 & 1,83 & 1,09 \\
4-ST & 207 & 11,7 & 9,0 & 5,7 & 20,0 & 34,7 & 2,4 & 5,0 & 17,0 & 1,52 & 1,19 \\
5-SC & 272 & 11,0 & 0,5 & 80,0 & 32,5 & 113,0 & 22,5 & 38,0 & 27,5 & 1,14 & 1,21 \\
\hline Serie $(n=20)$ & & & & & & & & & & \\
\hline Media & 164 & 10,5 & 7,9 & 20,4 & 24,4 & 52,6 & 6,3 & 8,7 & 43,4 & 2,68 & 1,19 \\
C.V.(\%) & 58,3 & 42,2 & 127,2 & 159,9 & 90,2 & 377,3 & 166,5 & 186,4 & 64,9 & 101,3 & 12,4 \\
\hline
\end{tabular}

Exp: exposición; Pen: pendiente; $\mathbf{L}+\mathrm{M}$ : liquen y musgo; Pl: plantas (pastos y arbustos); Hoj: hojarasca; Vtot: vegetación total; Aveg: altura de la vegetación; Pe: pedregosidad; Ps: profundidad del suelo; Hs: humedad del suelo $(0-3 \mathrm{~cm})$ y Den: densidad aparente.

Tabla 3. Características de las superficies en el período húmedo. Promedio por unidades y valor de la media y coeficiente de variación correspondientes al conjunto de la serie $(n=20)$.

\begin{tabular}{|cccccccccccc|}
\hline $\begin{array}{c}\text { Unidad } \\
(\%)\end{array}$ & $\begin{array}{c}\text { Exp } \\
(\%)\end{array}$ & $\begin{array}{c}\text { Pen } \\
(\%)\end{array}$ & $\begin{array}{c}\text { L+M } \\
(\%)\end{array}$ & $\begin{array}{c}\text { Pl } \\
(\%)\end{array}$ & $\begin{array}{c}\text { Hoj } \\
(\%)\end{array}$ & $\begin{array}{c}\text { Vtot } \\
(\%)\end{array}$ & $\begin{array}{c}\text { Aveg } \\
(\mathrm{cm})\end{array}$ & $\begin{array}{c}\text { Pe } \\
(\%)\end{array}$ & $\begin{array}{c}\text { Ps } \\
(\mathrm{cm})\end{array}$ & $\begin{array}{c}\text { Hs } \\
(\%)\end{array}$ & $\begin{array}{c}\text { Den } \\
\left(\mathrm{g} \mathrm{cm}^{-3}\right)\end{array}$ \\
\hline 1-HG & 131 & 12,0 & 9,1 & 68,4 & 1,22 & 78,8 & 3,7 & 5,2 & 36,8 & 28,0 & 1,21 \\
2-BG & 240 & 2,0 & 3,0 & 90,0 & 0,5 & 93,5 & 3,3 & 0 & 100 & 51,7 & 0,99 \\
3-TC & 72 & 11,3 & 1,3 & 51,5 & 18,8 & 71,7 & 3,7 & 1,8 & 43,3 & 53,4 & 0,89 \\
4-ST & 207 & 11,7 & 3,8 & 28,7 & 1,3 & 33,8 & 3,0 & 7,3 & 17,0 & 40,6 & 1,07 \\
5-SC & 272 & 11,0 & 6,0 & 70,0 & 2,8 & 78,8 & 27,5 & 12,3 & 27,5 & 30,6 & 1,17 \\
\hline Serie $(n=20)$ & & & & & & & & & & \\
\hline Media & 164 & 10,5 & 5,9 & 63,3 & 3,9 & 73,2 & 5,9 & 5,0 & 43,4 & 37,5 & 1,10 \\
C.V.(\%) & 58,3 & 54,7 & 146,9 & 43,2 & 203,5 & 33,7 & 130,2 & 120,7 & 64,9 & 41,8 & 21,0 \\
\hline
\end{tabular}

Exp: exposición; Pen: pendiente; L+M: liquen y musgo; Pl: plantas (pastos y arbustos); Hoj: hojarasca; Vtot: vegetación total; Aveg: altura de la vegetación; Pe: pedregosidad; Ps: profundidad del suelo; Hs: humedad del suelo $(0-3 \mathrm{~cm})$ y Den: densidad aparente. 
En los datos de densidad del suelo también es apreciable la diferencia entre las zonas de acumulación (BG) y el resto de las unidades, ya que en BG se observan los suelos más porosos y menos densos y consecuentemente con mayor capacidad de retención hídrica. No obstante, la densidad también es baja en las parcelas localizadas bajo las copas de Q. ilex subsp. ballota (TC) debido a una mayor concentración de materia orgánica en la superficie. Aunque las diferencias estacionales no han sido significativas, se han encontrado valores ligeramente más bajos en el período húmedo.

La pedregosidad de los suelos es generalmente baja. Las pequeñas diferencias detectadas entre el período seco y húmedo pueden explicarse por el lavado previo producido por escorrentía superficial durante las abundantes lluvias del invierno 1995-96. No obstante, en ambos periodos, la unidad con mayor pedregosidad es SC, con un $38 \%$ en el período seco y un $12,3 \%$ en el húmedo.

La humedad del suelo y la cubierta vegetal son las variables donde las diferencias estacionales y espaciales han sido más significativas. Durante el período seco, la humedad del suelo media, tras varios meses de precipitaciones escasas, es inferior al $3 \%$. No obstante se detecta una diferencia entre los fondos de valle $(8,1 \%)$ y el resto de las unidades $(<2 \%)$, debido a sus ya comentadas características físicas y topográficas. Estos datos contrastan notablemente con las mediciones de humedad efectuadas durante el período húmedo, con un valor medio próximo al $40 \%$, sobrepasándose en algunas unidades (BG y TC) el $50 \%$. El hecho de que en varias parcelas la humedad registrada al final del experimento sea igual a la inicial es indicador de las condiciones de suelos saturados en que se realizaron los experimentos en el período húmedo.

La vegetación total media es otro dato que marca considerablemente las diferentes condiciones antecedentes en que fueron realizados los experimentos. Así, en el período seco el porcentaje medio de vegetación total (liquen, musgo, plantas -herbáceas y arbustos- y hojarasca) fue de un 52,6\%. La variabilidad espacial es muy alta, siendo el coeficiente de variación (C.V.) del $377,3 \%$. Destaca en un nivel superior la unidad SC con un $113 \%$ de cobertura al superponerse las manchas de $L$. stoechas a un mantillo de hojarasca. En un nivel medio estarían las unidades BG y TC $(85,7$ y $70,3 \%$ respectivamente), aunque con una muy diferente componente en la cubierta, ya que en los fondos de valle (BG) la cubierta vegetal está constituida principalmente por plantas herbáceas, mientras que en TC se trata esencialmente de hojas secas sobre el suelo desnudo. La sequía y el sobrepastoreo inciden en los bajos valores de HG y ST con un 28,2 y un $34,7 \%$ respectivamente. En ambiente húmedo el porcentaje medio de vegetación total es notablemente superior $(73,2 \%)$ al del ambiente seco y espacialmente mucho más uniforme (C.V. $=33,7 \%$ ). Por unidades, con excepción de ST, los valores oscilan entre el 71,7 y el $93,5 \%$, siendo herbáceas y arbustos el componente predominante. En ST la ausencia de hojarasca, debido al lavado previo por escorrentía superficial, explica que el porcentaje de protección sea bajo con los consiguientes riesgos de pérdida de suelo. 


\subsection{Respuesta erosiva durante el período seco}

La concentración media de la serie es de $0,40 \mathrm{~g} \mathrm{l}^{-1}$ con un coeficiente de variación bajo $\left(28,7 \%\right.$ ) (tabla 4). Los datos medios de las unidades oscilan entre los $0,31 \mathrm{~g} \mathrm{l}^{-1}$ observados en los fondos de valle (BG) y los $0,45 \mathrm{~g} \mathrm{l}^{-1}$ en las veredas del ganado (ST) (tabla 5), donde en la microparcela ST2 se llegó a alcanzar el valor máximo de 0,65 $\mathrm{g}^{-1}$ (tabla 4). La mayor permanencia de la cubierta vegetal en los fondos de valle, debido a que en ellos la vegetación no se agosta hasta finales de julio y agosto por su mayor capacidad de retención hídrica (Bernet, 1995; Schnabel, 1995), explica que las escorrentías sean menores, así como la pérdida de suelo. Por otro lado, los altos valores de concentración de sedimentos en la unidad ST han de relacionarse directamente con la acción del ganado sobre el medio, ya que la remoción continua del

Tabla 4. Concentración de sedimentos, pérdida de suelo total y tendencia temporal de la concentración de sedimentos durante la simulación de lluvia (TSC), en las diferentes microparcelas estudiadas, durante los períodos seco y húmedo.

\begin{tabular}{|c|c|c|c|c|c|c|}
\hline \multicolumn{4}{|c|}{ Ambiente SECO } & \multicolumn{3}{|c|}{ Ambiente HÚMEDO } \\
\hline Parcela & $\begin{array}{c}\text { Con. Sed. } \\
\mathrm{g} \mathrm{I}^{-1}\end{array}$ & $\begin{array}{l}\text { Erosión } \\
\mathrm{g} \mathrm{m}^{-2} \mathrm{~h}^{-1}\end{array}$ & $\begin{array}{c}\text { TCS } \\
\% \\
\end{array}$ & $\begin{array}{c}\text { Con. Sed. } \\
\mathrm{g} \mathrm{l}^{-1}\end{array}$ & $\begin{array}{l}\text { Erosión } \\
\mathrm{g} \mathrm{m}^{-2} \mathrm{~h}^{-1}\end{array}$ & $\begin{array}{c}\mathrm{TCS} \\
\%\end{array}$ \\
\hline \multicolumn{7}{|c|}{ 1-Ladera con pastos } \\
\hline HGI & 0,34 & 12,35 & $-0,43$ & 0,21 & 9,60 & $-0,47$ \\
\hline HG2 & 0,64 & 7,15 & $-1,44$ & 0,22 & 8,94 & $-0,24$ \\
\hline HG3 & 0,50 & 12,13 & $-0,50$ & 0,26 & 10,58 & $-0,55$ \\
\hline HG4 & 0,41 & 5,41 & $-0,50$ & 0,12 & 3,90 & $+0,01$ \\
\hline HG5 & 0,47 & 14,73 & $-1,28$ & 0,29 & 13,64 & $-0,37$ \\
\hline HG6 & 0,29 & 6,34 & $-0,96$ & 0,18 & 7,30 & $-0,22$ \\
\hline HG7 & 0,35 & 8,30 & $-0,38$ & 0,25 & 9,72 & $-0,73$ \\
\hline HG8 & 0,28 & 10,37 & $-0,94$ & 0,21 & 8,02 & $-0,28$ \\
\hline HG9 & 0,51 & 7,87 & $-1,16$ & 0,18 & 5,56 & $-0,34$ \\
\hline \multicolumn{7}{|c|}{ 2-Fondos de valle con pastos } \\
\hline BG1 & 0,25 & 6,86 & $+0,02$ & 0,00 & 0,00 & 0,00 \\
\hline BG2 & 0,29 & 7,78 & $-0,80$ & 0,25 & 9,39 & $-1,22$ \\
\hline BG3 & 0,38 & 3,02 & $-0,34$ & 0,25 & 10,42 & $-0,76$ \\
\hline \multicolumn{7}{|c|}{ 3-Áreas bajo arbolado } \\
\hline TCl & 0,32 & 11,32 & $-0,85$ & 0,19 & 3,89 & $-0,15$ \\
\hline TC2 & 0,28 & 7,85 & $+0,23$ & 0,18 & 6,22 & $-0,56$ \\
\hline TC3 & 0,51 & 7,95 & $-1,26$ & 1,58 & 59,99 & $-2,87$ \\
\hline \multicolumn{7}{|c|}{ 4-Veredas } \\
\hline ST1 & 0,43 & 7,87 & $-1,22$ & 1,62 & 56,45 & $-3,96$ \\
\hline ST2 & 0,65 & 10,66 & $-2,44$ & 0,47 & 20,09 & $-1,99$ \\
\hline ST3 & 0,28 & 8,12 & $+0,67$ & 0,20 & 8,44 & $-0,09$ \\
\hline \multicolumn{7}{|c|}{ 5-Arbustos } \\
\hline SC1 & 0,39 & 4,34 & $-1,48$ & 0,25 & 11,45 & $-0,62$ \\
\hline $\mathrm{SC} 2$ & 0,42 & 5,96 & $-1,51$ & 0,10 & 1,02 & $-0,43$ \\
\hline Media & 0,40 & 8,32 & $-0,83$ & 0,35 & 13,93 & $-0,79$ \\
\hline $\mathrm{CV}(\%)$ & 28,73 & 33,68 & $-82,76$ & 120,75 & 112,75 & $-125,68$ \\
\hline
\end{tabular}


suelo crea una verdadera área fuente, con la producción permanente de material dispuesto a ser erosionado. En esta unidad, la cubierta de hojarasca es insuficiente para proteger el suelo de los procesos erosivos. Cerdà y Lavee (1995) han apreciado una relación positiva entre altas tasas de erosión y pastoreo, ya que este tipo de práctica ejerce un severo control sobre la vegetación, incrementa el porcentaje de suelo desnudo y remueve la costra superficial por pisoteo. El desbroce del suelo en zonas mediterráneas, con la supresión de la capa vegetal y remoción de los centímetros superficiales, ha supuesto un aumento de la erodibilidad 12 veces respecto a suelos no alterados (Cerdà, 1995b).

En general existe una ausencia total de relación entre cubierta vegetal y concentración de sedimentos (figura $2 \mathrm{a}$ ), como también puede deducirse del contraste entre una variabilidad espacial alta de la vegetación que sin embargo no se traduce en una también alta variabilidad espacial en la concentración de sedimentos. Sin embargo, sí se aprecia una cierta correlación negativa entre escorrentía generada y concentración de sedimentos, aunque su valor $p$ no sea significativo (figura $3 a$ ). El hecho de que las mayores escorrentías respondan a las concentraciones de sedimentos más bajas y que en la mayoría de las microparcelas la tendencia temporal de la concentración de sedimentos durante el experimento sea negativa demuestra que el material dispuesto a ser erosionado es limitado y que el flujo superficial generado es capaz de evacuar todo el material (Abrahams et al., 1988). Ello explica que no exista una relación estrecha entre concentración de sedimentos y erosión en los casos estudiados.

La erosión media de la serie fue de $8,32 \mathrm{~g} \mathrm{~m}^{-2} \mathrm{~h}^{-1} \mathrm{y}$, al igual que ocurría con la concentración de sedimentos, la variabilidad espacial es baja ( C.V. $=33,7 \%$ ). Siendo la concentración de sedimentos observada baja, dependiendo de la mayor o menor escorrentía fluctuarán las tasas de erosión (figura 4a). Si ST era la unidad con mayor concentración de sedimentos, sin embargo ocupa el tercer lugar en la pérdida total de suelo debido a su menor volumen de escorrentía como consecuencia de procesos de infiltración asociados a macroporos (tabla 5). La unidad con menores pérdi-
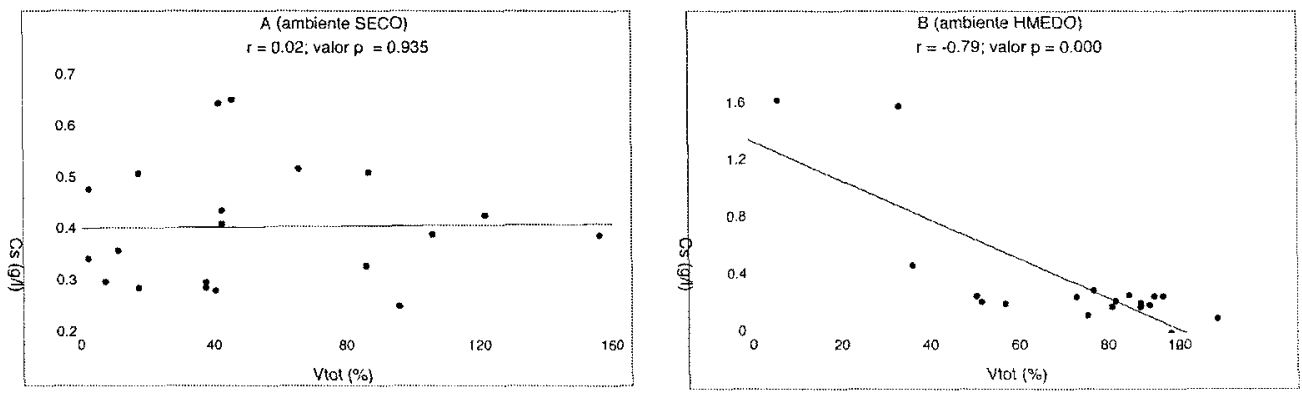

Figura 2. Correlación lineal entre vegetación total y concentración de sedimentos en ambiente seco (A) y en ambiente húmedo (B). 
das es SC $\left(5,15 \mathrm{~g} \mathrm{~m}^{-2} \mathrm{~h}^{-1}\right)$, a pesar de su concentración de sedimentos más bien alta $\left(0,40 \mathrm{~g} \mathrm{l}^{-1}\right)$, debido esencialmente al efecto pantalla del arbusto que intercepta gran parte de la lluvia y provoca altas tasas de infiltración a través de la porosidad asociada a su sistema radicular (Cerdà et al., 1998; Ceballos, 1999). La unidad HG registra la mayor tasa de erosión con $9,41 \mathrm{~g} \mathrm{~m}^{-2} \mathrm{~h}^{-1}$, ya que es la segunda unidad, tanto en el ranking de suelos susceptibles a ser erosionados como de producción de escorrentía.
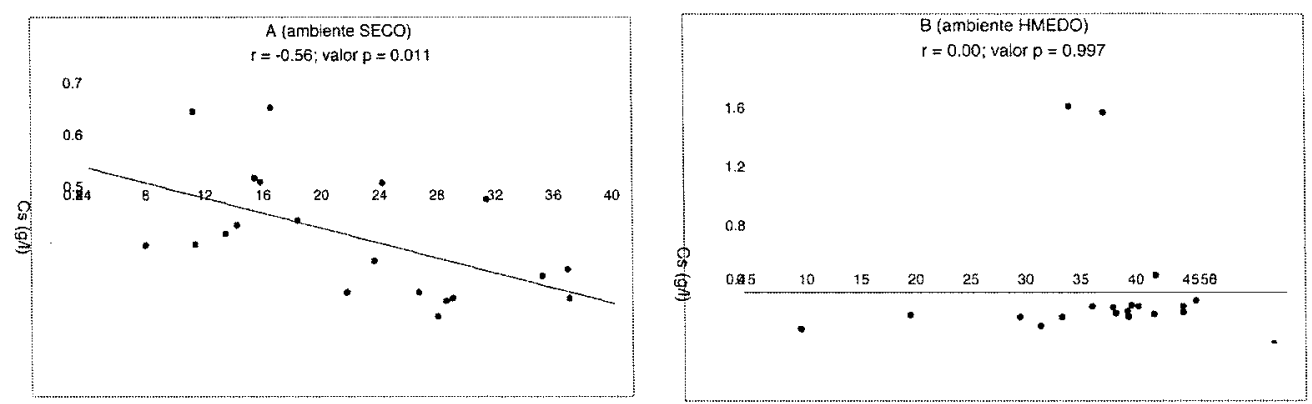

Figura 3. Correlación lineal entre escorrentía superficial y concentración de sedimentos en ambiente seco $(A)$ y en ambiente húmedo $(B)$.
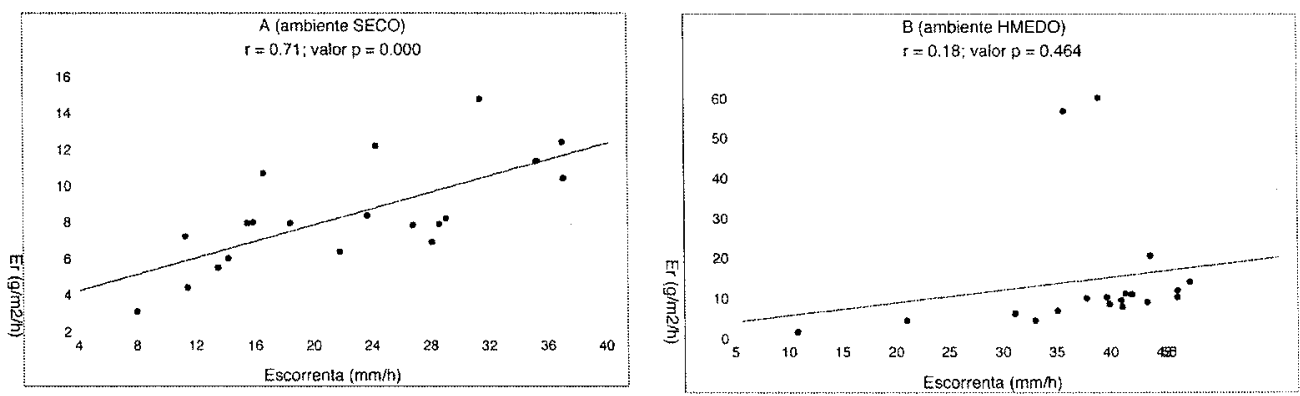

Figura 4. Correlación lineal entre escorrentía superficial y pérdida total de suelo durante las simulaciones en ambiente seco $(A)$ y en ambiente húmedo (B).

En la figura 5a se observa como las mayores pérdidas se producen en los suelos menos protegidos. Al no apreciarse ningún tipo de relación entre concentración de sedimentos y vegetación total, atribuimos la relación anterior a la existente entre suelo más desprotegido y mayores escorrentías. 

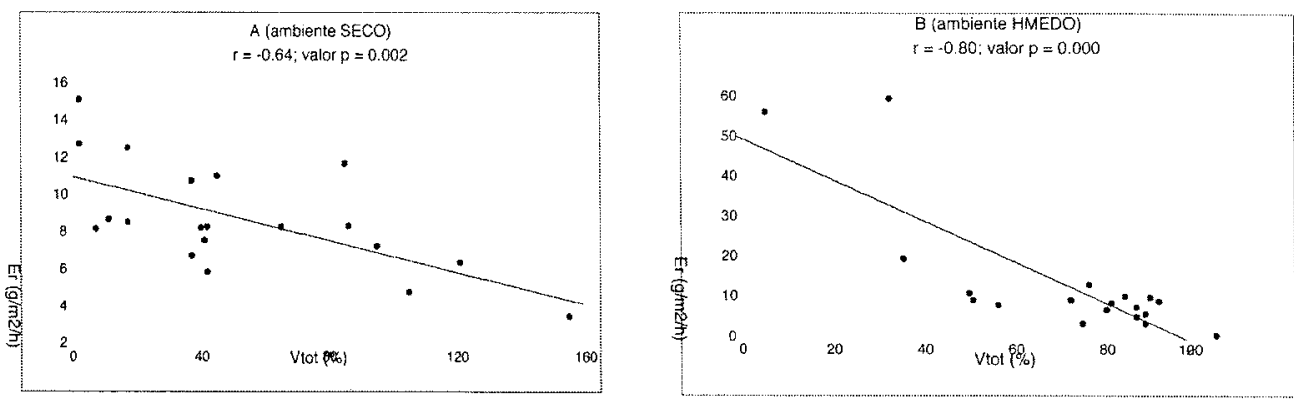

Figura 5. Correlación lineal entre vegetación total y pérdida total de suelo durante las simulaciones en ambiente seco (A) y en ambiente húmedo (B).

Tabla 5. Concentración de sedimentos, pérdida de suelo total, tendencia temporal de la concentración de sedimentos y escorrentía superficial en las diferentes unidades consideradas en ambiente seco. Datos medios y coeficientes de variación.

\begin{tabular}{|c|cc|cc|cc|cc|}
\hline \multirow{2}{*}{ Unidad } & \multicolumn{2}{|c|}{ Concentr. Sediment. } & \multicolumn{2}{|c|}{ Erosión } & \multicolumn{2}{|c|}{ Tend. Conc. Sed. } & \multicolumn{2}{c|}{ Escorrentía } \\
\cline { 2 - 9 } & $\mathrm{g} \mathrm{l}^{-1}$ & $\%$ & $\mathrm{~g} \mathrm{~m}^{-2} \mathrm{~h}^{-1}$ & $\%$ & $\%$ & $\%$ & $\mathrm{~mm} \mathrm{~h}^{-1}$ & $\%$ \\
\hline HG & 0,42 & 26,7 & 9,41 & 31,6 & $-0,84$ & $-44,9$ & 23,71 & 38,1 \\
BG & 0,31 & 18,4 & 5,88 & 35,0 & $-0,37$ & $-90,6$ & 20,70 & 43,9 \\
TC & 0,37 & 26,9 & 9,04 & 17,9 & $-0,63$ & $-99,8$ & 26,33 & 30,3 \\
ST & 0,45 & 33,4 & 8,89 & 14,2 & $-1,00$ & $-128,0$ & 21,15 & 25,8 \\
SC & 0,40 & 4,6 & 5,15 & 15,7 & $-1,49$ & $-0,80$ & 12,66 & 11,1 \\
\hline
\end{tabular}

\subsection{Respuesta erosiva durante el período húmedo}

En ambiente húmedo, salvo excepciones, la concentración de sedimentos en la escorrentía ha sido menor a causa de i) los mayores porcentajes de la cubierta total de plantas; ii) la ausencia parcial de ganado en la finca; iii) el lavado superficial previo por escorrentía, como consecuencia de las abundantes lluvias caídas entre noviembre de 1995 y el momento de realizarse los experimentos (febrero de 1996); y iv) los elevados coeficientes de escorrentía generados durante los propios experimentos de simulación, debido a la mínima capacidad de infiltración de los suelos saturados. En relación con ello, hay estudios que demuestran una relación exponencial positiva entre lavado superficial y la humedad antecedente (Luk, 1985; Luk y Hamilton, 1986). La concentración media de sedimentos fue de $0,35 \mathrm{~g} \mathrm{l}^{-1}$ con un C.V. muy alto $(120,7 \%)$ explicado por los valores extremos de las microparcelas TC3 y ST1, con 1,58 y 1,62 $\mathrm{g} \mathrm{l}^{-1}$ respectivamente, siendo precisamente las unidades ST y TC aquéllas con mayores concentraciones medias (tablas 4 y 6). Si excluyéramos del análisis estadístico las microparcelas TC3 y ST1, tanto la media como el coeficiente de variación decrecerían significativamente, con unos valores respectivos de $0,21 \mathrm{~g} \mathrm{l}^{-1}$ y $43 \%$. 
Tabla 6. Concentración de sedimentos, pérdida de suelo total, tendencia temporal de la concentración de sedimentos y escorrentía superficial en las diferentes unidades consideradas en ambiente húmedo. Datos medios y coeficientes de variación.

\begin{tabular}{|c|cc|cc|cc|cc|}
\hline \multirow{2}{*}{ Unidad } & \multicolumn{2}{|c|}{ Concentr. Sediment. } & \multicolumn{2}{|c|}{ Erosión } & \multicolumn{2}{c|}{ Tend. Conc. Sed. } & \multicolumn{2}{c|}{ Escorrentía } \\
\cline { 2 - 9 } & $\mathrm{g} \mathrm{I}^{-1}$ & $\%$ & $\mathrm{~g} \mathrm{~m}^{-2} \mathrm{~h}^{-1}$ & $\%$ & $\%$ & $\%$ & $\mathrm{~mm} \mathrm{~h}^{-1}$ & $\%$ \\
\hline HG & 0,21 & 22,3 & 8,58 & 31,3 & $-0,35$ & $-57,2$ & 39,24 & 12,7 \\
BG & 0,17 & 70,7 & 9,91 & 5,2 & $-0,66$ & $-76,3$ & 43,89 & 16,1 \\
TC & 0,65 & 100,9 & 23,37 & 110,9 & $-1,19$ & $-100,4$ & 30,86 & 24,7 \\
ST & 0,76 & 80,9 & 28,32 & 72,2 & $-2,01$ & $-78,4$ & 40,11 & 9,2 \\
SC & 0,18 & 43,2 & 6,23 & 83,6 & $-0,53$ & $-18,4$ & 45,32 & 27,7 \\
\hline
\end{tabular}

Los altos valores de TC3 y ST1 se explican por la acción del ganado. El pisoteo de las ovejas remueve el material superficial del suelo, destruyendo la costra superficial. Inicialmente hay disponible material para ser erosionado con las primeras escorrentías, pero se agota durante el desarrollo de la simulación, como se comprueba en que las tendencias de la concentración de sedimentos durante los experimentos en estas microparcelas sean marcadamente negativas en comparación con el resto del conjunto $(-2,87$ y $-3,96 \%$ respectivamente). Al igual que en el periodo seco, las menores concentraciones se miden en los fondos de valle con $0,17 \mathrm{~g}^{1^{-1}}$ de media.

Se aprecia una correlación significativa entre vegetación total y concentración de sedimentos, sobre todo porque las tres parcelas con mayores concentraciones se caracterizan por presentar una cubierta vegetal total inferior al $40 \%$ (figura $2 \mathrm{~b}$ ). Entre escorrentía superficial y concentración de sedimentos la relación es inexistente (figura 3b).

A pesar de que las concentraciones sean menores durante el período húmedo, sin embargo sus valores de erosión son más altos por las mayores escorrentías generadas. La media obtenida ha sido de $13,93 \mathrm{~g} \mathrm{~m}^{-2} \mathrm{~h}^{-1}$ con un C.V. muy alto $(112,7 \%)$ (tabla 4). Si excluimos de la serie las dos microparcelas excepcionales, los valores anteriores decrecen de forma apreciable $\left(8,72 \mathrm{~g} \mathrm{~m}^{-2} \mathrm{~h}^{-1}\right.$ de media y C.V. $\left.=47,7 \%\right)$.

Aunque no hayan tenido las mayores escorrentías, debido a su alta concentración de sedimentos, las unidades ST y TC son las que registran las mayores pérdidas totales (tabla 6). Si durante el período seco HG fue la unidad con mayor pérdida, ahora es la segunda unidad con menores pérdidas detrás de $\mathrm{SC}$, con tan sólo $8,58 \mathrm{~g} \mathrm{~m}^{-2} \mathrm{~h}^{-1}$.

Al igual que ocurría con la concentración de sedimentos, se aprecia una correlación significativa entre vegetación total y pérdida de suelo (figura 5 b) y una ausencia de relación con la escorrentía superficial (figura $4 \mathrm{~b}$ ).

De modo general, los resultados apuntan que las mayores concentraciones de sedimentos se localizan en aquellas zonas con suelos más desprotegidos, tanto por el efecto de la sequía como del tránsito del ganado, y que en la pérdida de suelo de Guadalperalón el control responde más al suministro de material que a la capacidad de transporte de la arroyada (Morgan, 1986). 
En el ecosistema dehesa las condiciones superficiales del suelo pueden variar sustancialmente, en un intervalo de tiempo relativamente corto, en relación con el clima y el uso del suelo. Los pastos en la dehesa se caracterizan por un marcado carácter estacional (Bernet, 1995; Schnabel, 1997), dependiendo, tanto su densidad como calidad, de la cantidad y distribución de las precipitaciones (Schnabel et al., 1996). Por otro lado, los ritmos de pastoreo inciden de manera considerable en los niveles de degradación del suelo, estando demostrada la relación existente entre intensificación del pastoreo, degradación del suelo, reducción de la biomasa, incremento de la superficie desnuda, y aumento de la producción de sedimentos (Warren et al., 1986; Weltz y Wood, 1986a y 1986b; Spaeth et al., 1996).

En ocasiones es difícil discernir si en la dehesa el incremento de los porcentajes de suelo desnudo, y por lo tanto de los riesgos erosivos, se deben en mayor o menor grado a la carencia de precipitaciones (sequía) o a la intensificación del uso del suelo (sobrepastoreo). En la dehesa estudiada, durante el año hidrológico 1994-95 la precipitación fue muy baja $(331,5 \mathrm{~mm})$ y la carga ganadera media $\left(1,6\right.$ ovejas ha $\left.{ }^{-1}\right)$. Ello se tradujo en que en septiembre de 1995 el porcentaje medio de suelo desnudo en las vertientes fue de un $56 \%$. El año siguiente, 1995-96, fue muy húmedo $(720,1$ $\mathrm{mm}) \mathrm{y}$, aunque en los tres primeros meses del año hidrológico la carga ganadera fue alta $\left(2,3\right.$ ovejas ha $\left.{ }^{-1}\right)$, las tres cuartas partes restantes del año se caracterizaron por un pastoreo muy controlado con secuencias alternativas de ausencia de ganado en la finca y presencia con intensidad media-baja ( 1 oveja ha ${ }^{-1}$ ). Consecuencia directa de un año con abundantes lluvias y baja presión ganadera fue que el porcentaje de suelo desnudo en septiembre de 1996 se redujo a un $32 \%$. El año 1996-97 fue también bastante húmedo $(699,4 \mathrm{~mm})$, pero con una presión ganadera intensa y permanente durante todo el tiempo $\left(2,4\right.$ ovejas ha $^{-1}$ y 0,1 vacas ha $\left.{ }^{-1}\right)$, lo cual provocó un altísimo porcentaje medio de suelo desnudo en septiembre de 1997 (73\%). Estos datos indican que, aunque las condiciones climáticas sean buenas para el desarrollo del estrato herbáceo (años 1995-96 y 1996-97), la intensidad del uso del suelo es determinante para explicar los niveles de degradación del mismo (año 1996-97).

Con el fin de comparar los resultados de erosión obtenidos durante los experimentos con datos medidos tras Iluvias reales en parcelas abiertas tipo Gerlach, la tabla 7 ofrece el balance de 4 eventos. Los eventos fechados los días 7 de agosto de 1992 y 24 de agosto de 1997 responcien a dos tormentas estivales de alta intensidad que provocaron unas pérdidas medias en vertientes de 107 y $77 \mathrm{~g} \mathrm{~m}^{-2}$ respectivamente. En ambos casos la humedad edáfica y el estado de degradación de la cubierta vegetal fueron similares a la serie de experimentos realizada en el periodo seco. Las notables diferencias respecto a los datos de los experimentos (media de $8,3 \mathrm{~g} \mathrm{~m}^{-2} \mathrm{~h}^{-1} \mathrm{y}$ valor máximo de $14,7 \mathrm{~g} \mathrm{~m}^{-2} \mathrm{~h}^{-1}$ en $\mathrm{HG}$ ) deben atribuirse al tamano pequeño de las microparcelas $\left(0,24 \mathrm{~m}^{2}\right)$, en comparación con el tamaño medio de las parcelas abiertas $\left(19 \mathrm{~m}^{2}\right)$, ya que esta circunstancia limita notablemente la capacidad erosiva del flujo superficial al no poder concentrarse de forma progresiva. Los datos de los eventos del 30 de diciembre de 1995 y 8 de enero de 1996 responden a unas condiciones similares a las de las simulaciones durante el período húmedo, ya que éstas fueron 
realizadas 4 semanas después. Al contrario que en condiciones secas, los valores medios de la tabla $\left(7,7\right.$ y $\left.6,6 \mathrm{~g} \mathrm{~m}^{-2}\right)$ están por debajo de los registrados en todas las unidades con excepción de SC. La interceptación de la energía cinética de la lluvia por parte de la cubierta vegetal, que también ralentiza la velocidad de la escorrentía superficial y dificulta la articulación de flujo concentrado, más el lavado superficial por escorrentías previas, explican este comportamiento.

Tabla 7. Pérdida de suelo media en vertientes medida en diferentes eventos con lluvias reales en la cuenca de Guadalperalón.

\begin{tabular}{|c|c|c|c|c|}
\hline Evento & $\begin{array}{c}\text { Condiciones } \\
\text { ambientales }\end{array}$ & $\begin{array}{c}\text { Precipitación } \\
(\mathrm{mm})\end{array}$ & $\begin{array}{c}30 \mathrm{~min} \text { int } \\
\left(\mathrm{mm} \mathrm{h}^{-1}\right)\end{array}$ & $\begin{array}{c}\text { Erosión } \\
\left(\mathrm{gr} \mathrm{m}^{-2}\right)\end{array}$ \\
\hline $07-08-1992$ & seca & 21,6 & 32,8 & 107,0 \\
$24-08-1997$ & seca & 70,0 & 40,0 & 76,8 \\
$30-12-1995$ & húmedas & 55,3 & 33,6 & 7,7 \\
$08-01-1996$ & húmedas & 34,8 & 20,4 & 6,5 \\
\hline
\end{tabular}

Tabla 8. Concentración de sedimentos (Cs) en diversas áreas mediterráneas obtenidas mediante simulación de lluvia.

\begin{tabular}{|llll|}
\hline Lugar & Uso del suelo & Cs $\left(g t^{1}\right)$ & Referencia \\
Valencia (España) & matorral & 0,73 & Cerdà et al. (1995) \\
Valencia (España) & área incendiada & 5,01 & Cerdà et al. (1995) \\
Valencia (España) & cortafuegos & 7,13 & Cerdà (1995a) \\
Valencia (España) & matorral & 0,40 & Cerdà (1994) \\
Alicante (España) & terrazas en badlands & 5,85 & Cerdà (1994) \\
Murcia (España) & campo abandonado-regenerado & 13,55 & Cerdà (1996) \\
Murcia (España) & campo abandonado-desnudo & 19,63 & Cerdà (1996) \\
Murcia (España) & espartizal & 1,87 & Cerdà (1996) \\
Murcia (Espanaa) & bosque de pinos & 0,39 & Cerdà (1996) \\
Desierto de Judea (Israel) & suelo desnudo & 2,31 & Cerdà (1996) \\
\hline
\end{tabular}

Finalmente, de modo general, como muestra la tabla 8 , las concentraciones obtenidas son muy bajas en comparación con los resultados registrados con metodología y equipo similares en varios ambientes mediterráneos, lo que supone que los suelos estudiados son poco erosionables. Ello también indica que la erodibilidad de un suelo no está relacionada con la escorrentía superficial, ya que en el estudio del comportamiento hidrológico de la dehesa a escala de pedón se ha constatado que las escorrentías en la dehesa son significativamente mayores que las de otros ambientes mediterráneos (Ceballos, 1999). No obstante, aunque las pérdidas registradas no parezcan importantes desde un punto de vista cuantitativo, sí lo son desde uno cualitativo, ya que afectan a la capa del suelo más rica en materia orgánica, y por la tanto más fértil y productiva, lo que supone un paulatino empobrecimiento ante la imposibiliclad de regeneración del horizonte orgá- 
nico. En varios puntos las muestras recogidas para la determinación de los sedimentos han mostrado un acusado tono turbio debido a la alta proporción de restos orgánicos, tanto de origen vegetal como animal, con la consiguiente pérdida de nutrientes.

\section{Conclusiones}

Dependiendo de la cantidad y distribución de las precipitaciones, así como de la intensidad de la carga ganadera, una de las características destacables de los ecosistemas adehesados es la variabilidad estacional de sus condiciones ambientales, siendo especialmente significativa en elementos como la humedad edáfica y la cubierta vegetal de la superficie del suelo.

A partir de los resultados obtenidos, con los experimentos de lluvia simulada, puede concluirse que en ambiente seco la susceptibilidad de los suelos a ser erosionados (expresada en la concentración de sedimentos), es superior que en ambiente húmedo ante su deterioro físico y menor cobertura superficial. El volumen de pérdida total de suelo durante los experimentos está controlado por la mayor o menor producción de escorrentía, circunstancia que explica que en ambiente húmedo, a pesar de las menores concentraciones de sedimentos, las pérdidas de suelo sean superiores a las registradas en el período seco.

La tendencia negativa de la concentración de sedimentos durante los experimentos indica que el control de la pérdida de suelo, en el ecosistema estudiado, responde más al suministro del material que a la capacidad de transporte por arroyada. Debido a ello, los resultados obtenidos, en comparación con otras zonas estudiadas con la misma metodología, muestran que las pérdidas de suelo en la dehesa son bajas. No obstante, debe matizarse la gran importancia de estas pérdidas desde un punto de vista cualitativo, ya que afectan al horizonte más fértil del perfil edáfico.

Espacialmente destaca el contraste entre las bajas pérdidas medidas en los fondos de valle, debido a su posición topográfica, alta capacidad de retención hídrica y perdurabilidad de la cubierta herbácea, y las zonas frecuentadas por el ganado, en donde la degradación de la cubierta vegetal es manifiesta y la remoción del material continua.

\section{Agradecimientos}

Este estudio ha sido financiado por la CICYT en el marco del proyecto AMB95/0986-CO2-02, y apoyado por la Junta de Extremadura, Dirección General de Enseñanzas Universitarias e Investigación. 


\section{6.- Bibliografía}

Abrahams, A.D.; Parsons, A.J. y Luk, S-H. (1988): Hydrologic and sediment responses to simulated rainfall on desert hillslopes in Southern Arizona. Catena, 15, p. 103-117.

Bernet, R. (1995): La cubierta berbácea en sistemas de dehesa degradados: conexiones entre vegetación y erosión. Cáceres, Fundicot-Extremadura, Universidad de Extremadura y Junta de Extremadura.

Ceballos, A. (1999): Balance de agua de una cuenca bidrográfica bajo explotación de dehesa en Extremadura. Madrid, Servicio de Publicaciones de la Universidad de Extremadura.

Ceballos, A. y Schnabel, S. (1998): Hydrological behaviour of a small catchment in the dehesa landuse system (Extremadura, SW Spain). Journal of Hydrology, 210, p. 146-160.

Cerdà, A. (1993): Metodologías para el estudio de la hidrología y erosión de superficies degradadas (badland) a partir de lluvia simulada. Cuaternario y Geomorfología, 7 , p. 35-48.

Cerdà, A. (1994): Arroyada superficial en terrazas de cultivo abandonadas. El caso del País Valenciano. Cuadernos de Geografia, 56, p. 135-154.

Cerdà, A. (1995a): Factores y variaciones espacio-temporales de la infiltración en los ecosistemas mediterráneos. Logroño, Editorial Geoforma.

Cerdá, A. (1995b): Hidrología y erosionabilidad de los suelos en ambientes semi-áridos, Petrer, Alicante. Studia Oecológica, 12 , p. $159-163$
Cerdà, A. (1996): Impacto del abandono del cultivo sobre la pérdicta de suelo y agua en un ambiente semiárido. Cuenca del río Guadalentín, Murcia. XIV Congreso Nacional de Geografia, p. 74-79.

Cerdà, A. y Lavee, H. (1995): Escorrentia y erosión en los suelos del desierto de Judea. Geograpbicalia, 32, p. 17-36.

Cerdà, A.; Imeson, A.C. y Calvo, A. (1995): Fire and aspect induced differences on the erodibility and hydrology of soils at La Costera, Valencia, southeast Spain. Catena, 24, p. 289-304.

Cerdà, A.; Ibánez, S. y Calvo, A. (1997): Design and operation of a small and portable rainfall simulator for rugged terrain. Soil Technology, 11, p. 163-170.

Cerdà, A.; Schnabel, S.; Ceballos, A. y GómezAmelia, D. (1998): Soil hydrological response under simulated rainfall in the Dehesa Ecosystem, Extremadura, Sw Spain. Earth Surface Processes and Landforms, 23, p. 195-209.

Epema, G.F. y Riezebos, H.TH. (1983): Fall velocity of waterdrops at different heights as a factor influencing erosivity of simulated rain. En De Ploey, J. (ed), Rainfall simulation, nunoff and soil erosion. Catena Supplement, 4, p. 2-17.

Gómez-Amelia, D. (1985): La penillanura cacereña. Estudio geomorfológico. Cáceres, Departamento de Geografía, Servicio de Publicaciones de la Universidad de Extremadura.

Gónez Amelia, D. y Schnabel, S. (1992): Procesos sedimentológicos e hidrológicos en una pequeña cuenca bajo explotación 
de dehesa en Extremadura. En López Bermúdez, F.; Conesa García, C. y Romero Díaz, M.A. (eds) II Reunión Nacional de Geomorfología. Murcia, S.E.G, p. 55-63.

Gómez Gutiérrez, J.M. (1987): El monte adehesado. Significación económica y ecológica actual. Revista de Estudios AgroSociales, 42, p. 171-193.

Ibáñez, J.J.; Recuero, M.A. y Vicente, A. (1981): Propuesta de ordenamiento de los agrosistemas de dehesa en la Península Ibérica. Revista de Estudios Agro-Sociales, 14, p. 39-79.

Luk, S.H. (1985): Effect of antecedent soil moisture content on rainwash erosion. Catena, 12, p. 129-139.

Luk, S.H. y Hamilton, H. (1986): Experimental effects of antecedent moisture and soil strength on rainwash erosion of two luvisols, Ontario. Geoderma, 37, p. 29-43.

Mateos, B. y Schnabel, S. (1998): Medición de la interceptación de las precipitaciones por la encina (Quercus rotundifolia Lam.): metodología y primeros resultados. En Gómez Ortiz, A., Salvador Franch, F., Schulte, L. y García Navarro, A. (eds) Investigaciones recientes de la Geomorfología española. Logroño, Geoforma Ediciones, p. 529-537.

Meyer, L.D. (1988): Rainfall simulators for soil conservation research. En Lal, R. (ed) Soil Erosion Research Methods. Soil and Water Conservation Society/ International Society of Soil Science, p. 74-95.

Morgan, R.P.C. (1986): Soil Erosion and Conservation. New York, Longman.
Schnabel, S. (1995): Hydrologische und erosive Prozesse in einem kleinen Einzugsgebiet unter silvo-pastoraler Landnutzung in Extremadura, Spanien. Freie Universität Berlin, tesis doctoral.

Schnabel, S. (1997): Soil erosion and nunoff production in a small watershed under silvopastoral landuse (dehesas) in Extremadura, Spain. Logroño, Geoforma Ediciones.

Schnabel, S.; Gómez Amelia, D. y Bernet, R. (1996): La pérdida de suelo y su relación con la cubierta vegetal en una zona de dehesa. En Campesino, A. y Velasco, C. (eds) VII Coloquio Ibérico de Geografia. Cáceres, Asociación de Geógrafos españoles, Dpto de Geografía y O.T. (UEX), p. 195-206.

Spaeth, K.E., Thurow, T.L., Blackburn, W.H. y Pierson. F.B. (1996): Ecological dynamics and management effects on rangeland hydrologic processes. En Spaeth, K.E., Pierson. F.B., Weltz, M.A. y Hendricks, G. (eds) Grazingland Hydrology Issues: Perspectives for the 21ts Century. Denver, Colorado, Society for Range management, p. 25-51.

United Nations (1977): Desertification: its causes and consequences. Oxford, Pergamon Press.

Warren, S.D.; Blackburn, W.H. y Taylor, J.R. (1986): Effects of season and stage of rotation cycle on hydrologic condition of rageland under intensive rotation grazing. Journal of Range management, 39(6), p. 486-490.

Weltz, M. y Wood, M.K. (1986a): Short duration grazing in Central New Mexico: Effects on sediment production. Journal of Soil and Water Conservation, 41(4), p. 262-266.

Weltz, M. y Wood, M.K. (1986b): Short duration grazing in Central New Mexico: Effects on infiltration rates. Journal of Range management, 39(4), p. 365-368. 